\title{
Study of interface asymmetry in InAs-GaSb heterojunctions
}

\author{
M. W. Wang, D. A. Collins, and T. C. McGill \\ T. J. Watson, Sr. Laboratory of Applied Physics, California Institute of Technology, \\ Pasadena, California 91125 \\ R. W. Grant \\ Rockwell Science Center, Thousand Oaks, California 91360 \\ R. M. Feenstra \\ IBM Research Division, T. J. Watson Research Center, Yorktown Heights, New York 10598
}

(Received 8 February 1995; accepted 25 March 1995)

\begin{abstract}
We present reflection high energy electron diffraction, secondary ion mass spectroscopy, scanning tunneling microscopy and x-ray photoelectron spectroscopy studies of the abruptness of InAs-GaSb interfaces. We find that the interface abruptness depends on growth order: InAs grown on GaSb is extended, while GaSb grown on InAs is more abrupt. We first present observations of the interfacial asymmetry, including measurements of band alignments as a function of growth order. We then examine more detailed studies of the InAs-GaSb interface to determine the mechanisms causing the extended interface. Our results show that $\mathrm{Sb}$ incorporation into the InAs overlayer and As exchange for $\mathrm{Sb}$ in the $\mathrm{GaSb}$ underlayer are the most likely causes of the interfacial asymmetry. (C) 1995 American Vacuum Society.
\end{abstract}

\section{INTRODUCTION}

The arsenide-antimonide system has a number of technologically interesting applications, such as high speed InAsAlSb oscillators, ${ }^{1}$ novel InAs-AlSb-GaSb based ${ }^{2-4}$ and InAs- $-\mathrm{GaSb}^{5}$ tunnel structures and InAs- $\mathrm{Ga}_{1-x} \mathrm{In}_{x} \mathrm{Sb}$ infrared superlattice detectors. ${ }^{6,7}$ Interfacial properties are very important in these applications; however, the mixed anion nature of the arsenide-antimonide system makes control of these properties particularly difficult.

Recent studies have shown that the electrical and structural properties of InAs-GaSb interfaces depend on the order in which they are grown. ${ }^{8-10}$ In those studies, cross sectional scanning tunneling spectroscopy (STS) and scanning tunneling microscopy (STM) were used to examine a series of InAs-GaSb superlattices. It was found that interfaces were rougher for InAs-grown-on-GaSb (InAs/GaSb) than for GaSb-grown-on-InAs (GaSb/InAs); this was true for both the InSb-like and GaAs-like interface bonding arrangements.

In this article, we use reflection high energy electron diffraction (RHEED), secondary ion mass spectroscopy (SIMS), STM and x-ray photoelectron spectroscopy (XPS) to study this observed growth order dependence. We show that while the GaSb/InAs interface is abrupt, there is significant anion intermixing at the InAs/GaSb interface. This intermixing exists both in the form of $\mathrm{Sb}$ riding up and incorporating into InAs overlayers and As exchanging for $\mathrm{Sb}$ in $\mathrm{GaSb}$ underlayers.

\section{EXPERIMENT}

All of the crystals reported here were grown in a PerkinElmer 430 molecular beam epitaxy (MBE) system equipped with cracked $\mathrm{Sb}$ and As sources. Unless noted otherwise, the substrate temperature was adjusted to be roughly $5{ }^{\circ} \mathrm{C}$ below the point at which $\mathrm{GaSb}$ in a cracked $\mathrm{Sb}$ flux undergoes a transition from a $1 \times 3$ to a $1 \times 5$ surface reconstruction $\left(T_{\text {sub }} \sim 380^{\circ} \mathrm{C}\right)$. It has been shown that the presence of this
$1 \times 5$ reconstruction is crucial to inhibit background As incorporation in the antimonide layers of $\operatorname{InAs}-\mathrm{Ga}_{x} \mathrm{In}_{1-x} \mathrm{Sb}$ superlattices. $^{11}$

Details of the experimental procedures used to obtain the STM, RHEED and XPS data are given in Refs. 8, 12 and 13, respectively. The SIMS measurements were performed by Charles Evans and Associates on a CAMECA IMS-4f machine using $3.0 \mathrm{keV} \mathrm{O}_{2}^{+}$ions.

\section{OBSERVATION OF INTERFACIAL ASYMMETRY}

In addition to the structural asymmetry observed by STM, mentioned in the Introduction, evidence for chemical asymmetry in the InAs-GaSb system has been observed by XPS. The XPS studies consisted of determining elemental peak intensity ratios for a number of $\mathrm{InAs}-\mathrm{GaSb}$ heterojunctions grown under a variety of growth conditions. Figure 1 shows two representative XPS scans of $20 \AA$ of GaSb grown on InAs and $20 \AA$ of InAs grown on GaSb. Core-level peak intensities and binding energies were obtained by performing peak isolation, integrated background subtraction and fitting the peaks to Voigt functions, as detailed in Ref. 13. To minimize electron diffraction effects, the escape orientation of the photoelectrons was kept constant from sample to sample. Core-level peak intensity ratios were then determined for each sample. Using this procedure, peak intensity ratios from different XPS scans on the same sample were reproducible to better than $2 \%$.

Since photoelectron escape depths are not known with certainty, it was not possible to determine the interfacial abruptness from a single XPS measurement. Instead, comparisons between a number of samples were required in order to conclude the existence of interfacial asymmetry. The basic results from this study are summarized in Table I, which shows the range of measured XPS peak intensity ratios for both InAs/GaSb and GaSb/InAs structures. Both interface types (InSb-like and GaAs-like) were studied for each 


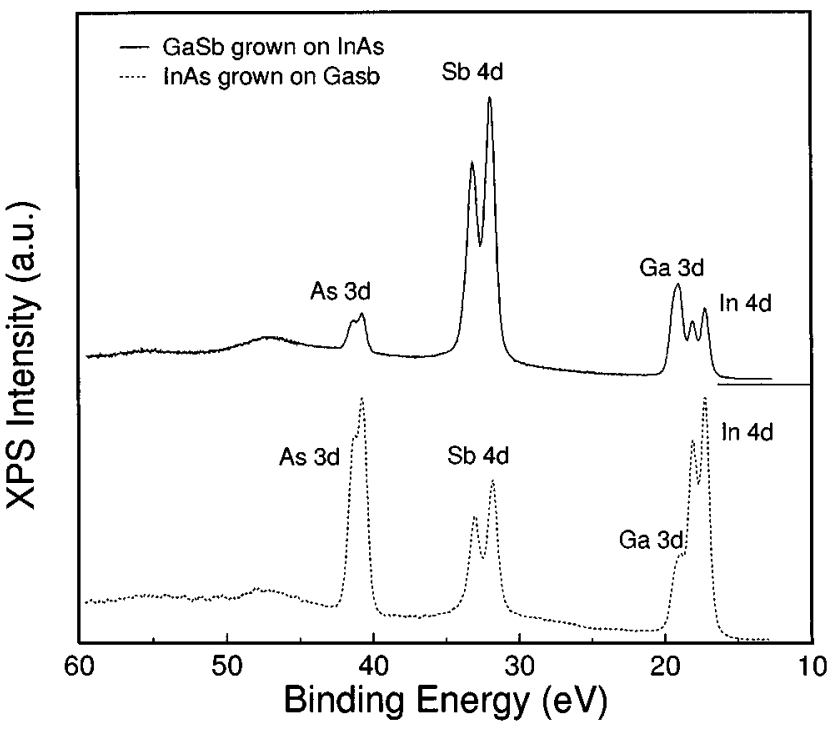

FIG. 1. Representative XPS scans of GaSb/InAs and InAs/GaSb heterostructures. The two data sets are offset for clarity.

growth order and are included in the ranges shown. The notation $x g: y s$ specifies $x$ growths and $y$ XPS scans used to obtain a given set of values. Note that only ratios between elements primarily on the same side of the interface are used, since ratios between elements on opposing sides of the interface are very sensitive to fluctuations in overlayer thickness.

Table I shows that the range in the measured peak intensity ratios for the InAs/GaSb samples is much larger than that for the $\mathrm{GaSb} / \mathrm{InAs}$ samples. The results for the $\mathrm{GaSb} /$ InAs samples are consistent with abrupt interfaces, where the small range in values can be explained by changes in interface composition (InSb-like or GaSb-like). However, the range of values for the InAs/GaSb samples is too large to be due to a change in interface composition, and can only be explained by an extended InAs/GaSb interface. At the growth temperature employed, the bulk diffusion constants of the group III atoms are extremely small, so the extended InAs/GaSb interface is most likely due to intermixing of the anion sublattice. A more detailed analysis, showing comparisons between experimental and calculated peak intensity ratios supports this explanation. ${ }^{14}$

The interfacial asymmetry at InAs-GaSb interfaces was also observed in XPS studies of the band alignments in this system. Results from this study showed no dependence of

TABLE I. Measured variation in XPS peak intensity ratios, for both InAs/ $\mathrm{GaSb}$ and $\mathrm{GaSb} / \mathrm{InAs}$ growth orders. The notation $x g$ : $y s$ specifies $x$ growths and $y$ XPS scans used to obtain a given set of values. Both interface types (InSb-like and GaAs-like) were studied for each growth order. The nominal overlayer thickness was $\sim 20 \AA$.

\begin{tabular}{ccc}
\hline \hline & \multicolumn{2}{c}{ Measured range of XPS peak area ratios } \\
\cline { 2 - 3 } Structure & $\mathrm{Sb}_{4 d} / \mathrm{Ga}_{3 d}$ & $\mathrm{As}_{3 d} / \mathrm{In}_{4 d}$ \\
\hline $\begin{array}{c}\mathrm{GaSb} / \mathrm{InAs} \\
4 g: 7 s\end{array}$ & $1.20-1.31$ & $0.63-0.86$ \\
$\mathrm{InAs} / \mathrm{GaSb}$ & $0.55-1.24$ & $0.81-1.37$ \\
$12 g: 17 s$ & \\
\hline
\end{tabular}

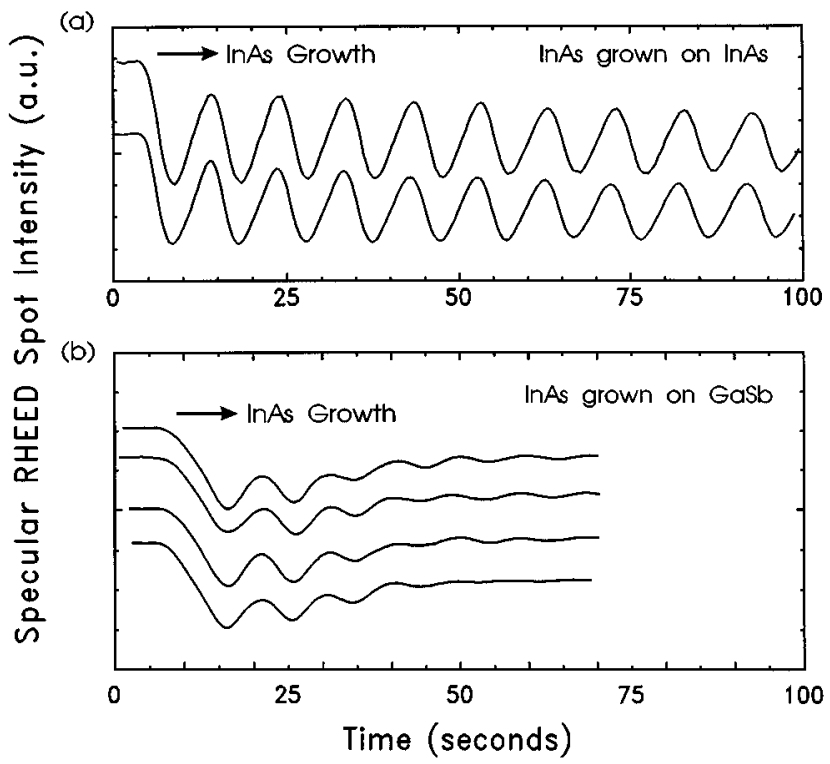

FIG. 2. Plots of the specular RHEED spot intensity during the growth of InAs on (a) InAs and (b) GaSb. The difference in the RHEED intensity indicates a different growth mode for InAs grown on GaSb compared to InAs grown on InAs.

the valence band offset on interface composition; however, a $90 \mathrm{meV}$ increase in the valence band offset was observed for InAs/GaSb compared to $\mathrm{GaSb} / \mathrm{InAs}$, with the variation in the values greater for InAs/GaSb compared to $\mathrm{GaSb} / \mathrm{InAs} .{ }^{15}$ The $90 \mathrm{meV}$ increase in the valence band offset for InAs/GaSb was attributed to the extended nature of this interface. It should be noted that this result is consistent with STS observations of a larger density of states in the conduction band of InAs layers near InAs/GaSb interfaces as compared to $\mathrm{GaSb} /$ InAs interfaces. ${ }^{8,9}$ The observed $90 \mathrm{meV}$ larger valence band offset for the InAs/GaSb growth order is another indication of the asymmetry in InAs-GaSb interfaces.

In the following sections we present studies which reveal the growth mechanisms leading to the extended InAs/GaSb interface. The intermixing of the anion sublattice can take the form of either $\mathrm{Sb}$ riding into InAs overlayers or As incorporation in GaSb underlayers. These two possibilities are examined below.

\section{Sb RIDING UP INTO InAs OVERLAYERS}

\section{A. RHEED studies}

Previously reported RHEED studies have noted unusual behavior for InAs growth on GaSb. ${ }^{12}$ In particular, the RHEED reconstruction of the InAs near the InAs/GaSb interface shows an uncharacteristic $2 \times 3$ or $1 \times 3$ surface reconstruction. Only after deposition of 2 to 4 monolayers of InAs does the $1 \times 1$ reconstruction characteristic for the given growth conditions appear. The $2 \times 3(1 \times 3)$ surface reconstruction is consistent with Sb riding up into InAs overlayers, since we have also observed this surface reconstruction for $\operatorname{InAs}_{x} \mathrm{Sb}_{1-x}$ alloys.

Studies of RHEED oscillations also show unusual behavior at InAs/GaSb interfaces. Figure 2 shows specular RHEED spot intensity oscillations during the growth of InAs 
on InAs [Fig. 2(a)] and GaSb [Fig. 2(b)], under nominally identical growth conditions. In the first case, the RHEED intensity profile shows a classic oscillatory behavior bounded by a slowly decaying envelope function. In contrast, the RHEED profile for InAs grown on GaSb behaves quite differently, with a change in the envelope function after a few monolayers of growth, corresponding to the transition in the RHEED reconstruction pattern. The absence of such unusual behavior for $\mathrm{GaSb} / \mathrm{InAs}$ growths indicates a clear interfacial asymmetry. A detailed qualitative analysis of the RHEED data ${ }^{14}$ has indicated that this asymmetry is most likely due to an extended anion sublattice for the InAs/GaSb growth order.

\section{B. XPS studies}

To confirm that $\mathrm{Sb}$ does ride into the InAs layers, XPS spectra for $20 \AA \mathrm{InAs} / \mathrm{GaSb}$ heterostructures were compared to bulk InAs and GaSb spectra. This analysis showed an increase in the $\mathrm{Sb} 4 d$ to $\mathrm{Ga} 3 d$ peak intensity ratio for the $\mathrm{InAs} / \mathrm{GaSb}$ sample relative to a $1 \times 5$ reconstructed $\mathrm{GaSb}$ standard. If we assume a fixed cation sublattice, the only explanation for this increase is $\mathrm{Sb}$ behaving like a surfactant and riding up into the InAs overlayer. This is also consistent with an observed small decrease in the As $3 d$ to In $4 d$ peak intensity ratio relative to bulk InAs. Quantitative estimates of the relative amounts of $\mathrm{Sb}$ incorporating in and riding through the InAs layer are difficult because of the large variation in published photoelectron escape depths. However, $\mathrm{Sb}$ was observed to ride through $1500 \AA$ thick layers of InAs; hence we can conclude that at least some of the $\mathrm{Sb}$ is on the InAs surface with the additional possibility of $\mathrm{Sb}$ incorporation within the InAs.

To determine the source of $\mathrm{Sb}$ atoms, GaSb surfaces with both $1 \times 3$ and $1 \times 5$ surface reconstructions were studied. The results showed roughly $25 \%$ to $50 \%$ of a monolayer of excess $\mathrm{Sb}$ for the $1 \times 5$ reconstructed GaSb. Also, previous studies of $\mathrm{Sb}$ exposures of InAs surfaces showed evidence for metallic $\mathrm{Sb}$ island formation. ${ }^{13}$ Both experiments indicate the existence of excess $\mathrm{Sb}$ on $1 \times 5 \mathrm{GaSb}$ surfaces prior to InAs growth. This excess $\mathrm{Sb}$, as well as any $\mathrm{Sb}$ liberated from the $\mathrm{GaSb}$ underlayer by an As-for-Sb exchange reaction, is the probable source for interdiffused $\mathrm{Sb}$ atoms.

\section{SIMS studies}

While both the RHEED and XPS studies described above suggest that $\mathrm{Sb}$ incorporates into the InAs overlayer, the data are not conclusive. To confirm the existence of Sb incorporation, two InAs/GaSb interfaces were studied using SIMS: one grown at $380{ }^{\circ} \mathrm{C}$ and the other at $475{ }^{\circ} \mathrm{C}$ (see Fig. 3). Based on the larger InAs bond strength compared to InSb, Sb incorporation, if it exists, should be greater for the lower growth temperature. Thus, a comparison of the SIMS profiles should determine whether or not $\mathrm{Sb}$ is incorporating into the InAs. Superposition of the curves was performed by a least squares minimization of the difference between the two profiles. The solid curve in Fig. 3 is for the near-surface interface grown at $380{ }^{\circ} \mathrm{C}$, while the dashed curve is for the near-substrate interface grown at $475{ }^{\circ} \mathrm{C}$. It is evident that the near-surface interface has a more extended $\mathrm{Sb}$ profile,

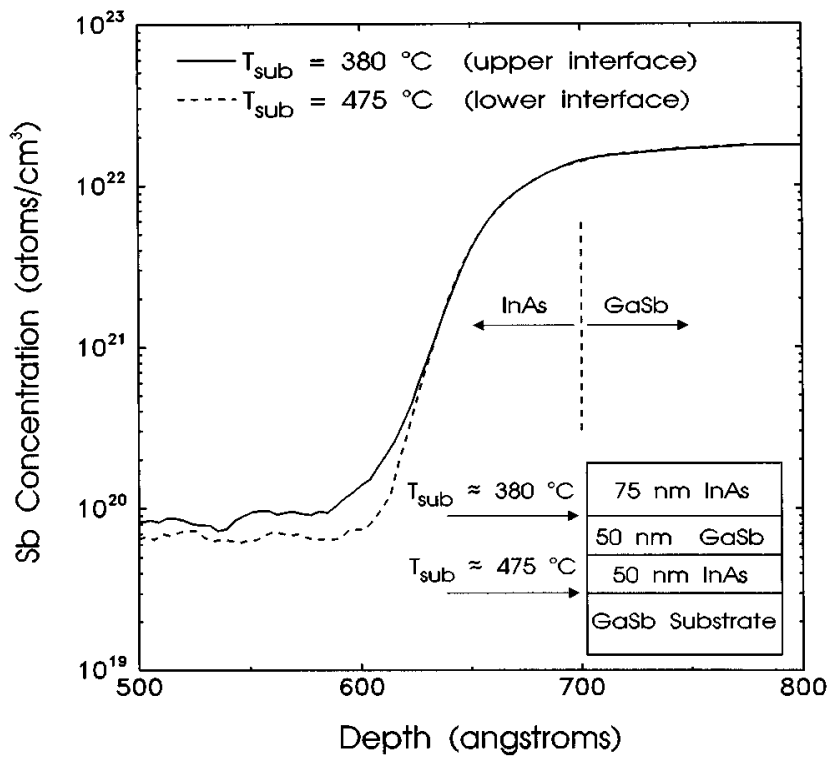

FIG. 3. Sb profile measured by SIMS for two InAs-grown-on-GaSb interfaces grown at substrate temperatures of $475{ }^{\circ} \mathrm{C}$ (dashed line) and $380{ }^{\circ} \mathrm{C}$ (solid line).

most apparent at $\sim 600 \AA$. This is significant, since any sputter roughening, to which $\mathrm{GaSb}$ is particularly susceptible ${ }^{16}$ will be more pronounced at the near-substrate interface. If the higher temperature InAs/GaSb interface is abrupt, then Fig. 3 indicates roughly two percent Sb incorporation in InAs near the interface over a length scale of a few tens of $\AA$. Of course, if the high-temperature grown $\mathrm{InAs} / \mathrm{GaSb}$ interface is not abrupt, the amount of Sb incorporation at the lower temperature will be even greater. Thus, the SIMS analysis shows conclusively that in InAs/GaSb interfaces, $\mathrm{Sb}$ from the underlying $\mathrm{GaSb}$ can incorporate into the InAs overlayer and that the amount of incorporation is sensitive to the growth temperature. This effect was reproduced in two separate SIMS analysis of the same sample. This result has also been independently confirmed by recent cross sectional STM measurements. ${ }^{17}$

\section{As INCORPORATION IN GaSb UNDERLAYERS}

\section{A. XPS studies}

To investigate the possibility of As incorporation in $\mathrm{GaSb}$ underlayers, we studied the effects of exposing a bulk $\mathrm{GaSb}$ surface to a cracked As flux as a function of soak time. The purpose of mimicking the interface formation process in this manner was to determine whether or not As-for-Sb exchange occurs during the formation of $\mathrm{InAs} / \mathrm{GaSb}$ interfaces. For both the XPS and RHEED exchange reaction studies, the As flux was reduced by a factor of 5-6 compared to typical growth fluxes in order to better time resolve the exchange reaction. The substrate temperature was kept at nominal growth temperature. Figure 4(a) shows a clear increase in the As $3 d$ to $\mathrm{Ga} 3 d$ peak intensity ratio as a function of soak time. To rule out the possibility of As deposition versus exchange, we also studied the Ga $3 p$ to Ga $3 d$ and $\mathrm{Sb} 4 d$ to $\mathrm{Ga}$ $3 d$ peak intensity ratios, shown in Fig. 4(b). Since the binding energies of Ga $3 p$ and $\mathrm{Ga} 3 d$ core levels differ by about 


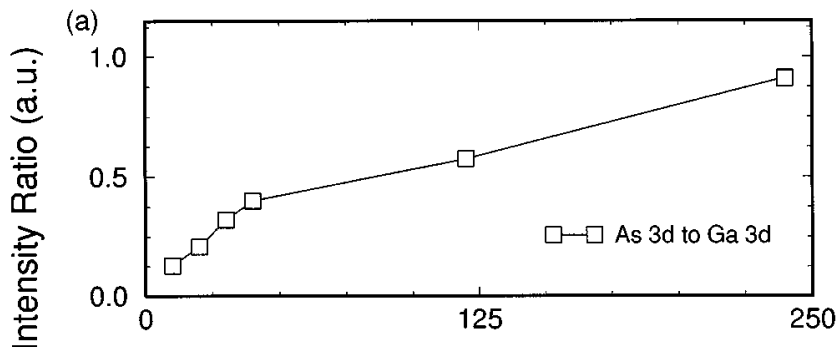

(b)

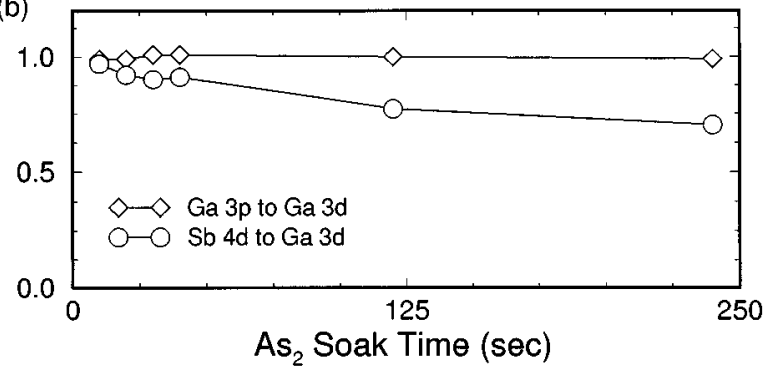

FIG. 4. XPS peak intensity ratios for $\mathrm{As}_{2}$ exposures of GaSb surfaces. (a) The nonsaturating As $3 d$ signal indicates either As-for-Sb exchange into the bulk GaSb, or As deposition. (b) The constant Ga $3 p$ to Ga $3 d$ peak intensity ratio and the decreasing $\mathrm{Sb} 4 d$ signal show that the increasing As $3 d$ signal is due to As-for-Sb exchange and not As deposition.

$90 \mathrm{eV}$, their escape depths will also differ. This results in a reduction in the $\mathrm{Ga} 3 p$ to $\mathrm{Ga} 3 d$ peak intensity ratio if an overlayer exists. Thus, the lack of a change in the Ga $3 p$ to Ga $3 d$ ratio indicates that substantial As deposition is not occurring during the exposure of GaSb to As. Also, the $\mathrm{Sb} 4 d$ to $\mathrm{Ga} 3 d$ ratio falls as the As exposure time increases. This strongly supports the hypothesis of an As-for-Sb exchange during the As exposure. Analysis of the As $3 d$ to $\mathrm{Ga} 3 d$ ratios using a range of escape depths indicates not only a surface As-for-Sb exchange reaction but also an additional bulk exchange. The point at which the slope in Fig. 4(a) changes ( $\sim 40$ seconds) corresponds rather well to the As $3 d$ to Ga $3 d$ signal expected from a surface As-for-Sb reaction. The slower increase after this point is consistent with a slower, bulk As-for-Sb exchange reaction. Further studies of InAs/ $\mathrm{GaSb}$ heterostructures also provide strong evidence for an As-for-Sb exchange reaction. ${ }^{14}$

\section{B. RHEED studies}

Examination of RHEED patterns during As soaks of GaSb also yields results consistent with a bulk As-for-Sb exchange. For roughly 30 seconds after the As soak is initiated, the RHEED reconstruction does not change, as expected, since $\mathrm{GaAs}$ and $\mathrm{GaSb}$ have the same surface reconstructions at the substrate temperature used $\left(\sim 380^{\circ} \mathrm{C}\right)$. However, the intensity of the RHEED specular spot decreases, indicating a disordered surface. Additionally, for roughly 3 minutes, the spacing between the specular and first order streaks does not change, indicating that the lattice constant of the surface does not change. As the exposure time increases, the RHEED reconstruction and streak spacing are observed to change, and eventually the pattern becomes spotty. It is interesting to note that the RHEED streak spacing stays constant even after XPS and STM data indicate that the bulk As-for-Sb ex-
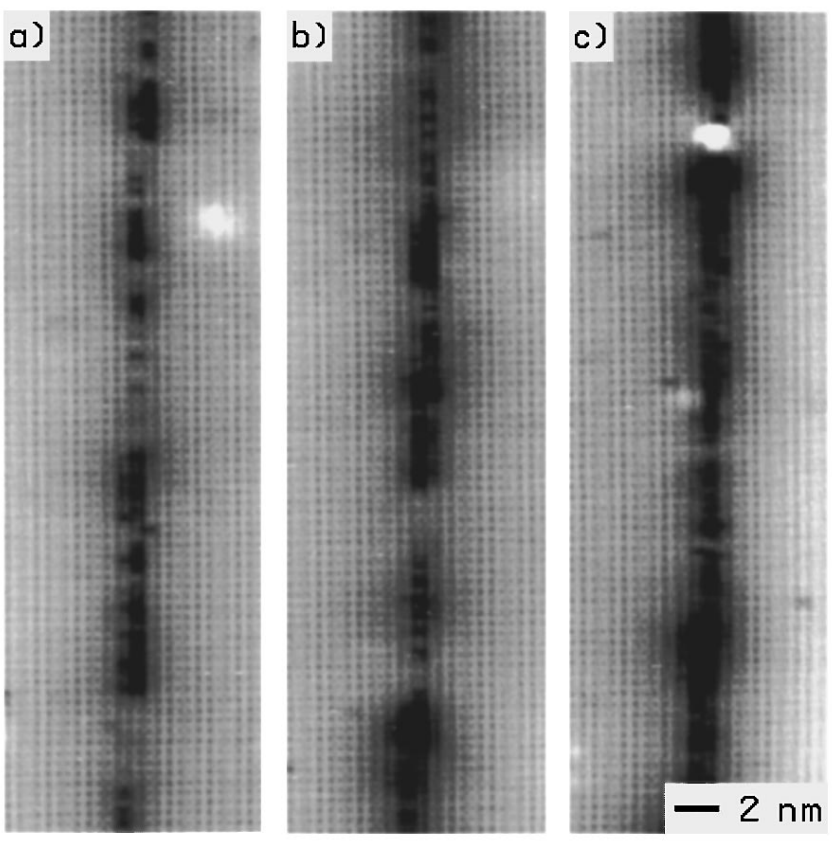

FIG. 5. Cross sectional STM images of the effect of exposing a GaSb surface to an As flux. Relative fluxes are 1, 1.5 and 3.0 for (a), (b), and (c), respectively. Anion states on the [110] surface are imaged (sample voltage -1.6 V). Gray-scale range is $1.0 \AA$. Growth direction is to the left.

change has begun to occur. This suggests that the bulk exchange results in a GaAs surface layer, which remains coherently strained until the strain energy is enough to generate dislocations.

\section{STM studies}

As further evidence of the As-for-Sb exchange reaction, Fig. 5 shows cross sectional STM images of bulk GaSb layers deposited on (100) GaSb wafers. This figure shows anion states on a [110] plane, hence the fringes in the images correspond to unit cells with a spacing of $6.095 \AA$. During growth of the GaSb epilayer, deposition was interrupted and the $\mathrm{GaSb}$ growth front was exposed to a cracked As flux for 3 seconds, with relative fluxes of $1,1.5$ and 3.0 for Figs. $5(\mathrm{a}), 5(\mathrm{~b})$, and 5(c), respectively. The dark patches in the STM images indicate reduced tunneling current due to the formation of GaAs, which has a lower valence band edge than $\mathrm{GaSb}$, during the As exposure. These patches are 1 to 2 unit cells deep and a few tens of $\AA$ across for Fig. 5(a) and 2 to 4 unit cells deep and nearly continuous in Fig. 5(c). The more extensive GaAs layers formed as a result of the higher As fluxes indicate that the surface and bulk As-for-Sb exchange reaction rates are not kinetically limited, but are limited by the As arrival rate. The GaAs layer formed this way seems to be coherently strained to the surrounding $\mathrm{GaSb}$ with no evidence of dislocation formation. However, it is extremely rough and non-uniform, indicating that any interface formation scheme which exposes a GaSb underlayer to an As flux will result in poor interface quality. 


\section{DISCUSSION}

In the preceding section we determined that the InAs/ $\mathrm{GaSb}$ interface is more extended than the GaSb/InAs interface due to $\mathrm{Sb}$ incorporation into the InAs overlayer and As-for-Sb exchange in the GaSb underlayer. In this section we describe possible driving forces for this interface asymmetry.

The explanation for the observed $\mathrm{Sb}$ incorporation into InAs is based on the surfactant nature of $\mathrm{Sb}$, which causes $\mathrm{Sb}$ to collect on the growth front of the GaSb underlayer. This $\mathrm{Sb}$ is then available to ride up into the InAs overlayer. Although $\mathrm{Sb}$ incorporation into InAs is not energetically favorable, entropy and the low growth temperature will drive some $\mathrm{Sb}$-for-As exchange, resulting in the observed $\mathrm{Sb}$ incorporation. In contrast, the As-for-Sb exchange in the $\mathrm{GaSb}$ underlayer is driven by the much larger bond strength of GaAs compared to GaSb. This process is also aided by the tendency of InAs to nucleate in islands which exposes the $\mathrm{GaSb}$ underlayer to an As flux during interface formation.

With these forces driving anion intermixing, obtaining an abrupt InAs/GaSb interface will be difficult. This occurs because reduction of the As-for-Sb exchange in $\mathrm{GaSb}$ requires that the equilibrium GaAs phase be suppressed, while reduction of $\mathrm{Sb}$ incorporation in InAs requires that the equilibrium InAs phase be enhanced. Thus, growth conditions which reduce $\mathrm{Sb}$ incorporation will tend to increase the As exchange and vice versa. In spite of this difficulty, initial studies have shown some improvement in the abruptness of InAs/GaSb interfaces under certain growth conditions. ${ }^{14}$

For $\mathrm{GaSb} / \mathrm{InAs}$ growth, the driving forces work towards an abrupt interface. First, As is not a surfactant at the growth temperatures employed; hence there is no tendency for it to accumulate on the InAs growth front where it could subsequently incorporate in GaSb overlayers. Second, since InAs is more stable than InSb, Sb-for-As exchange reactions during the nucleation of $\mathrm{GaSb}$ on InAs do not continue into the bulk InAs, but instead saturate after exchange of one monolayer of As. ${ }^{13}$

\section{SUMMARY}

We have successfully used RHEED, SIMS, STM and XPS to study the interfacial asymmetry in InAs-GaSb heterointerfaces. Significant anion intermixing is observed at the InAs/GaSb interface, while the $\mathrm{GaSb} / \mathrm{InAs}$ interface is found to be more abrupt. The intermixing exists both in the form of $\mathrm{Sb}$ riding up and incorporating into InAs overlayers, and as As-for-Sb exchange in GaSb underlayers during interface formation. This result is due to two fundamental material properties of the system. First, $\mathrm{Sb}$ is a surfactant at the growth conditions employed and consequently is available to ride up into InAs layers grown on GaSb. Second, the much greater bond strength of GaAs compared to GaSb drives an As-for-Sb exchange reaction into the GaSb underlayer.

\section{ACKNOWLEDGMENTS}

This work was supported by the Air Force Office of Scientific Research and the Office of Naval Research under Contract Nos. AFOSR-F49620-93-1-0258 and N00014-93-10881, respectively.

${ }^{1}$ E. R. Brown, J. R. Söderström, C. D. Parker, L. J. Mahoney, K. M. Molvar, and T. C. McGill, Appl. Phys. Lett. 58, 2291 (1991).

${ }^{2}$ M. Sweeny and J. Xu, Appl. Phys. Lett. 54, 546 (1989).

${ }^{3}$ J. R. Söderström, D. H. Chow, and T. C. McGill, Appl. Phys. Lett. 55, 1094 (1989).

${ }^{4}$ L. F. Luo, R. Beresford, and W. I. Wang, Appl. Phys. Lett. 55, 2023 (1989).

${ }^{5}$ D. A. Collins, E. T. Yu, Y. Rajakarunanayake, J. R. Söderström, D. Z.-Y. Ting, D. H. Chow, and T. C. McGill, Appl. Phys. Lett. 57, 683 (1990).

${ }^{6}$ D. L. Smith and C. Mailhiot, J. Appl. Phys. 62, 2545 (1987).

${ }^{7}$ R. H. Miles, D. H. Chow, J. N. Schulman, and T. C. McGill, Appl. Phys. Lett. 57, 801 (1990).

${ }^{8}$ R. M. Feenstra, D. A. Collins, D. Z.-Y. Ting, M. W. Wang, and T. C. McGill, Phys. Rev. Lett. 72, 2749 (1994).

${ }^{9}$ R. M. Feenstra, D. A. Collins, D. Z.-Y. Ting, M. W. Wang, and T. C. McGill, J. Vac. Sci. Technol. B 12, 2592 (1994).

${ }^{10}$ A. Y. Lew, E. T. Yu, D. H. Chow, and R. H. Miles, Appl. Phys. Lett. 65, 201 (1994).

${ }^{11}$ D. H. Chow, R. H. Miles, J. R. Söderström, and T. C. McGill, Appl. Phys. Lett. 56, 1418 (1990).

${ }^{12}$ D. A. Collins, T. C. Fu, D. H. Chow, and T. C. McGill, J. Vac. Sci. Technol. B 10, 1779 (1992).

${ }^{13}$ M. W. Wang, D. A. Collins, T. C. McGill, and R. W. Grant, J. Vac. Sci. Technol. B 11, 1418 (1993).

${ }^{14}$ D. A. Collins, M. W. Wang, R. W. Grant, R. M. Feenstra, and T. C. McGill (unpublished).

${ }^{15}$ M. W. Wang, D. A. Collins, R. W. Grant, R. M. Feenstra, and T. C. McGill, Appl. Phys. Lett. 66, 2981 (1995).

${ }^{16}$ M. Gauneau, R. Champlain, A. Rupert, Y. Toudic, R. Callec, and E. Andre, J. Appl. Phys. 73, 2051 (1993).

${ }^{17}$ R. M. Feenstra, D. A. Collins, and T. C. McGill, Superlatt. Microstruct. 15, 215 (1994). 